\title{
Markovian Random Walks on Square Lattice with Constant Non-Symmetric Diffusion Coefficients
}

\author{
Kosuke Hijikata, Ihor Lubashevsky, ${ }^{*}$ Alexander Vazhenin \\ University of Aizu, Tsuruga, Ikki-machi, Aizu-Wakamatsu City, Fukushima 965-8560, Japan \\ *E-mail for correspondence: i-lubash@u-aizu.ac.jp
}

\begin{abstract}
Within the rigor typical for physical models a new type non-symmetric diffusion problem is considered and the corresponding Brownian motion implementing such diffusion processes is constructed. As a particular example, random walks with internal causality on a square lattice are studied in detail. By construction, one elementary step of a random walker on the lattice may consist of its two succeeding jumps to the nearest neighboring nodes along the $x$ - and then $y$-axis or the $y$ and then $x$-axis ordered, e.g., clock-wise. It is essential that the second fragment of elementary step is caused by the first one, meaning that the second fragment can arise only if the first one has been implemented, but not vice versa. In particular, if for some reasons the second fragment is blocked, the first one may be not affected, whereas if the first fragment is blocked, the second one cannot be implemented in any case. As demonstrated, on time scales much larger then the duration of one elementary step these random walks are characterized by a diffusion matrix with non-zero anti-symmetric component, which is also justified by numerical simulation.
\end{abstract}

\section{Introduction}

The present paper poses a fundamental question about the completeness of the modern formalism of describing stochastic processes based on the FokkerPlanck equation and stochastic differential equations. The main attention is focused on the symmetry properties of the diffusion tensor $D_{i j}$ as a characteristic feature reflecting the premises of this formalism. In particular, within its frameworks the diffusion tensor must be symmetric. Leaping ahead, we note that there can be constructed a certain type of random walks for which the diffusion tensor $D_{i j}$ is not symmetric or, speaking more strictly, the diffusion tensor relating the diffusion flux with the gradient of the probability distribution function does not coincide with the diffusion tensor entering the Fokker-Planck equation. The first tensor may be non-symmetric and for the constructed random walks it is the case, whereas the second one is just its symmetric component. Because the boundary conditions that must be imposed on the Fokker-Planck equation deal with the diffusion flux, the possible non-symmetry of diffusion tensor is an essential property. The ob- tained results allow us to state that a new formalism should be developed to calculate directly the diffusion flux and, thus, to find an expression for the diffusion tensor different from its standard definition appealing to the walker displacement correlations.

The paper is organized as follows. Section 1.1 discusses some examples of physical systems, where a nonsymmetric diffusion tensor appears and their relation with the problem at hand. Section 1.2 presents short introduction to the used formalism of describing stochastic processes analyzed in this paper. Section 2 specifies the proposed model of random walks with a nonsymmetric diffusion tensor, the final section is Conclusion.

\subsection{Physical Systems with Non-Symmetric Diffusion}

It is well known that in semiconductors kept in a magnetic field $\mathbf{H}$ the diffusion of electrons or holes in one of the transverse directions, e.g., along an axis $\mathbf{i}$ $(\mathbf{i} \perp \mathbf{H})$, causes their transport in the other direction $\mathbf{j}=\mathbf{i} \times \mathbf{H} / H$ transverse to the field $\mathbf{H}$ and the axis $\mathbf{i}$ as well. In particular, this property is behind the photomagnetoelectric effect observed for the first time by Kikoin \& Noskov in 1933 [1] (for a detailed review see $[2])$. Namely, when a semiconductor plate placed in a magnetic field $\mathbf{H}$ as shown in Fig. 1 is illuminated by a beam of light perpendicular to its plane the electronhole pairs generated near the illuminated side start to diffuse towards the opposite side (in the direction $\mathbf{i}$ ). As a result, along the axis $\mathbf{j}$ a certain electric field is produced with strength $E \propto H$ proportional to the magnetic field. Via the Lorentz force, the regular drift of charged particles along the axis $\mathbf{i}$ caused by the gradient $\partial_{i} n$ of their concentration $n$ in this direction induces the particle diffusion flux $Q_{j}$ along the axis $\mathbf{j}$. Their relationship is of the form

$$
Q_{j}=-D_{j i} \partial_{i} n
$$

where the coefficient $D_{j i}$ can be regarded as an antisymmetric diffusion tensor, $D_{i j}=-D_{j i}[3]$.

Spatial diffusion of cosmic rays in turbulent magnetic fields is also described by anisotropic diffusion tensor $D_{i j}$ with an antisymmetric component [4]. The corresponding governing equation for the distribution $n$ of 

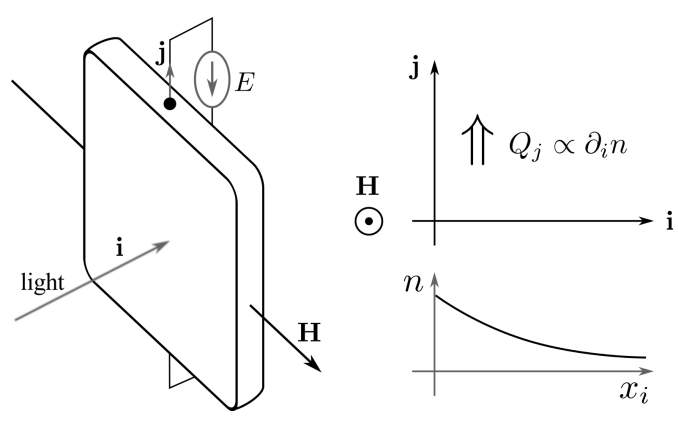

Fig. 1: Illustration of the photomagnetoelectric effect.

charged particles is usually written in the form

$$
\partial_{t} n=\sum_{i, j} \partial_{i}\left[D_{i j}(\mathbf{x}, t) \partial_{j} n\right]+\text { non-diffusion terms }
$$

where generally the tensor $D_{i j}(\mathbf{x}, t)$ depends on the spatial coordinates $\mathbf{x}$ and time $t$ as well. Markov chains described by equations similar to Eq. (2), where the tensor $D_{i j}$ is not symmetric $\left(D_{i j} \neq D_{j i}\right)$ and the non-diffusion terms are absent, are also referred to as the nonsymmetric diffusion processes (see, e.g., [5, 6] and references therein). In fact, such equations may be treated as the Fokker-Planck equation with the drift term aggregated into the effective diffusion tensor $D_{i j}(\mathbf{x})$. Due to the diffusion tensor $D_{i j}(\mathbf{x})$ depending on the spatial coordinates $\mathbf{x}$, its possible non-symmetry can be responsible for macroscopic effects.

It should be noted that the description of the photomagnetoelectric effect does not belong to the given class of non-symmetric diffusion processes because the diffusion tensor $D_{i j}$ introduced via relationship (1) between the diffusion flux and the particle gradient is constant and, so, its asymmetric components do not contribute to Eq. (2). In the photomagnetoelectric effect the appearance of electric field is due to the semiconductor plate boundaries blocking the motion of electrons and holes in the direction $\mathbf{j}$. The theory of the photomagnetoelectric effect [3] posits Exp. (1) appealing to the kinetic theory dealing with the motion of charged particles in magnetic fields and their scattering, e.g., by impurities in semiconductors. So, strictly speaking, the question about what stochastic process similar to simple Brownian motion of particles without inertia (simple random walks in physical space) can reproduce the photomagnetoelectric effect is beyond the developed theory.

The purpose of the present paper is to consider Markovian random walks characterized by constant kinetic coefficients that require the introduction of nonsymmetric diffusion tensor and, so, may be regarded as a distinct type of non-symmetric diffusion processes. However, before passing directly to this problem, it is worthwhile to remind some basic features in describing continuous Markovian stochastic processes.

\subsection{Continuous Markovian Random Walks and Their Description}

Let us consider Markovian processes that can be mimicked in terms of continuous in space and time Brownian random works in a certain $N$-dimensional phase space $\mathbb{R}^{N}$. Among various approaches to describing such random walks we note, first, the formalism of stochastic differential equations written as

$$
d \mathbf{x}=\mathbf{V}(\mathbf{x}, t) d t+\mathbf{S} d \mathbf{W}
$$

where $\mathbf{x}=\left\{x_{i}\right\}_{i=1}^{i=N} \in \mathbb{R}^{N}$ is a point of the given phase space, $\mathbf{V}=\left\{V_{i}\right\}$ is the velocity of the regular drift in this space, $\mathbf{W}=\left\{W_{\alpha}(t)\right\}_{\alpha=1}^{\alpha=M}$ is a collection of $M$ mutually independent Wiener processes, and the matrix $\mathbf{S}=\left\|S_{i \alpha}\right\|$ specifies the intensity of the corresponding Langevin forces. Keeping in mind the subject under consideration the intensity matrix $\mathbf{S}$ is assumed to be constant, which allows us not to distinguish between possible interpretations of the stochastic differential equation (3) because all of them are equivalent in this case. Equation (3) actually relates the walker displacement $d \mathbf{x}$ within one elementary (infinitesimal) step of duration $d t$ to its regular drift velocity $\mathbf{V}(\mathbf{x}, t)$ at a given point $\mathbf{x}$ and time $t$ and the random variable $\mathbf{S} d \mathbf{W} \propto \sqrt{d t}$ caused by the Langevin forces. Within the rigor of physical constructions we may interpret the solution of Eq. (3) for a given implementation of Wiener processes as the infinite sequence of elementary walker jumps (steps) in the phase space (Fig. 2).

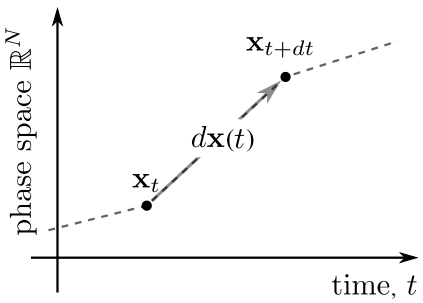

Fig. 2: Illustration of a walker trajectory generated by Eq. (3).

It should be noted that the question about the details of the walker motion between the initial $\mathbf{x}_{t}$ and terminal $\mathbf{x}_{t+d t}$ points of one elementary step is beyond this formalism which, actually, specifies the probabilistic properties of the collection of points $\left\{\mathbf{x}_{t}\right\}$. Nevertheless, when the matrix $\mathbf{S}(\mathbf{x})$ of the Langevin force intensity depends on the spatial coordinates $\mathbf{x}$, the introduction of some intermediate points $\chi\left(\mathbf{x}_{t}, \mathbf{x}_{t+d t}\right)$ can be justified in characterizing the walker motion within one elementary step. In constructing the solution of a stochastic differential equation similar to Eq. (3) the matrix $\mathbf{S}(\mathbf{x})$ should be taken at the points $\chi\left(\mathbf{x}_{t}, \mathbf{x}_{t+d t}\right)$ and the particular details of $\boldsymbol{\chi}\left(\mathbf{x}_{t}, \mathbf{x}_{t+d t}\right)$ determine the type of stochastic differential equation, exemplified by the Itô or Stratonovich interpretations [7].

The second approach, we note in the present paper, is the formalism of partial differential equations exem- 
plified by the forward Fokker-Planck equation

$$
\partial_{t} G=\sum_{i=1}^{N} \partial_{i}\left\{\sum_{j=1}^{N} \partial_{j}\left[D_{i j} G\right]-V_{i}(\mathbf{x}, t) G\right\}
$$

subject to the initial condition

$$
\left.G\left(\mathbf{x}, t \mid \mathbf{x}_{0}, t_{0}\right)\right|_{t=t_{0}}=\delta\left(\mathbf{x}-\mathbf{x}_{0}\right),
$$

where $G(\mathbf{x}, t)$ is the probability density of funding a walker at point $\mathbf{x}$ at time $t$ provided initially, $t=t_{0}$, it was located at the point $\mathbf{x}_{0}$. Here the diffusion matrix (tensor) $\mathbf{D}=\left\|D_{i j}\right\|$ is related to the matrix $\mathbf{S}$ of the Langevin force intensity by the relation

$$
D_{i j}=\frac{1}{2} \sum_{\alpha=1}^{M} S_{i \alpha} S_{j \alpha}
$$

which holds also in the case of the matrix $\mathbf{S}(\mathbf{x})$ depending on the spatial coordinates. Actually Exp. (5) stems from the definition of the diffusion coefficients

$$
D_{i j}(\mathbf{x}, t)=\lim _{d \tau \rightarrow 0} \frac{\left\langle\left(x_{i, d t+t}-x_{i, t}\right)\left(x_{j, d t+t}-x_{j, t}\right)\right\rangle}{2 d \tau}
$$

accepted in many textbooks (see, e.g., $[8,9]$ ) and implying the diffusion matrix $\mathbf{D}=\left\|D_{i j}\right\|$ to be symmetric. Due to the form of the Fokker-Planck equation (4) holding also for the diffusion matrix depending on time and spatial coordinates any antisymmetric component $D_{i j}^{a}$ $\left(D_{i j}^{a}=-D_{j i}^{a}\right)$ can be formally added to $\mathbf{D}$,

$$
D_{i j} \Rightarrow D_{i j}+D_{i j}^{a},
$$

because this replacement does not contribute to the form of the Fokker-Planck equation.

The probability (diffusion) flux $\mathbf{Q}=\left\{Q_{i}\right\}$ is introduced by appealing to a certain analogy of Brownian particle motion assuming such a particle to move along a smooth trajectory between the initial and terminal points of one elementary step shown in Fig. 2. Equivalently the Fokker-Planck equation (4) can be interpreted as the continuity equation for diffusing particles

$$
\partial_{t} G+\sum_{i=1}^{N} \partial_{i} Q_{i}=0
$$

and the expression

$$
Q_{i}=-\sum_{j=1}^{N} \partial_{j}\left[D_{i j} G\right]+V_{i}(\mathbf{x}, t) G,
$$

holding also for $\mathbf{D}=\mathbf{D}(\mathbf{x}, t)$, is employed in order to relate the probability flux $\mathbf{Q}=\left\{Q_{i}\right\}$ with the local characteristics of random walks (see, e.g., [9]). Naturally, here replacement (7) cannot be used with an arbitrary antisymmetric component $\left\|D_{i j}^{a}\right\|$.
If for some reasons the regular drift velocity can be written in the form

$$
V_{i}=\sum_{j=1}^{N} \partial_{j} D_{i j}(\mathbf{x}, t),
$$

where $\left\|D_{i j}(\mathbf{x}, t)\right\|$ is not a symmetric matrix, than via the corresponding replacement (7) the Fokker-Planck equation is reduced to

$$
\partial_{t} G=\sum_{i, j=1}^{N} \partial_{i}\left[D_{i j}(\mathbf{x}, t) \partial_{j} G\right]
$$

and the probability flux is given by the expression

$$
Q_{i}=-\sum_{j=1}^{N} D_{i j}(\mathbf{x}, t) \partial_{j} G .
$$

Exactly it is the case categorized as the non-symmetric diffusion problem noted above, where it becomes possible to introduce the notion of non-symmetric diffusion tensor within the classical approach to modeling Brownian motion. However, if the regular drift velocity and the diffusion matrix are constant this interpretation does not hold.

There is another way to fix a possible antisymmetric component $D_{i j}^{a}$ of diffusion matrix. It is to employ the boundary conditions that should be imposed on the corresponding stochastic differential equation and the Fokker-Planck equation depending on the physical properties of the system boundary. The corresponding relationship between the diffusion flux and the gradient of the probability density taken at the boundary can determine the non-symmetric diffusion tensor. There is a long-term history of studding such conditions for multi-dimensional diffusion processes starting from the series of seminal works including ones by Wentzell [10], Sato \& Ueno [11], Hiroshi [12], Watanabe [13], Skorokhod [14], for a detailed review see also monographs by Ikeda \& Watanabe [15] and Schuss [8]. We also note an approach to deriving the boundary conditions starting from the Chapman-Kolmogorov equation and employing the fact that a system boundary breaks the symmetry of random walker steps, giving rise to boundary singularities in the Kramers-Moyal expansion. Eliminating these singularities gives rise to the required boundary conditions [16].

By present paper we intend to attract attention to a distinct way of introducing the non-symmetric diffusion matrix. It is not related to the properties of system boundaries; the boundary to be considered below is merely reflecting, i.e., it just does not allow a walker to leave the region of random walks. Namely, this approach appeals to rotational type regularities of walker motion within one elementary step; as far as the translational regularities are concerned with, the random walks to be considered are purely symmetric and, so, their regular drift velocity is equal to zero. 


\section{Brownian Random Walks with Internal Causality}

In this Section we consider discrete random walks on a square lattice of spacing $a$, assuming that by one elementary step of duration $\tau$ a walker hops to one of the neighboring lattice nodes. The properties of these walker jumps are explained in Figs. 3-5. Such discrete random walks on lattices admit the continuous description on scales $t \gg \tau$, which enables the introduction of a diffusion matrix and a drift velocity to describe them.

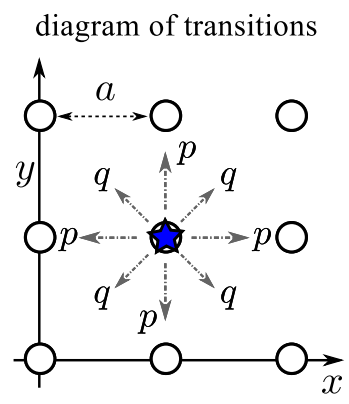

$$
p=\frac{(1-\epsilon)}{4}, \quad q=\frac{\epsilon}{4}
$$

Fig. 3: The analyzed random walks on the square lattice: The diagram showing possible transitions of the walker within one elementary step and their probabilistic weights

Figure 3 illustrates the main characteristics of random walks treated in the conventional way. Namely, it shows the neighboring nodes accessible for a walker by one jump and the corresponding probabilities of hopping to them. They are the four nearest neighbors gotten by the walker with the individual probability $p=(1-$ $\epsilon) / 4$, where $0 \leq \epsilon \leq 1$ is a given system parameter. The four nodes of the next shell of nearest neighbors are also acceptable with the individual probability $q=$ $\epsilon / 4$. These transitions specify directly the probabilistic properties of one elementary step treated as whole, in particular, the regular drift velocity of these random walks is equal to zero, $V_{x}=V_{y}=0$, and the diffusion matrix $\mathbf{D}^{s}$ characterizing them on time scales $t \gg \tau$ is symmetric (moreover, diagonal) with the components

$$
D_{x x}^{s}=D_{y y}^{s}=D^{s}=\frac{(1+\epsilon) a^{2}}{4 \tau}, D_{x y}^{s}=D_{y x}^{s}=0 .
$$

Here the subscript $s$ is used to underline the fact that exactly this matrix enters the corresponding FokkerPlanck equation and formally obeys definition (6).

Figure 4 shows all the eight implementations of one jump which actually mimics the effect of magnetic field normal to the plane of the given lattice. To avoid possible misunderstanding it should be noted that such random walks do not admit the introduction of regular walker velocity, thereby, the Lorenz force effect is reduced to some regular rotation within one elementary step. Namely, the proposed model assumes the following. When a walker randomly hops, for example, upward the axis $y$ (jump $\mathbf{j}_{+}$) and gets the upper nearest node, it either remains at this node with the probability $(1-\epsilon)$ or immediately hops (jump $\left.\mathbf{i}_{-}\right)$to the left nearest node with the probability $\epsilon$. These composed jumps implementations of one elementary jump

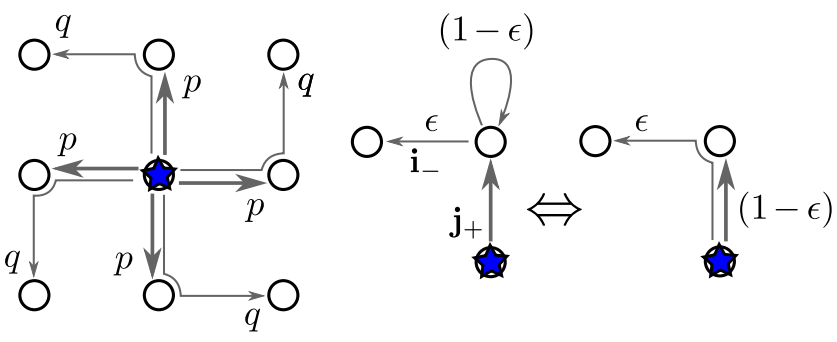

Fig. 4: The analyzed random walks on the square lattice: The possible implementations of one elementary step.

effect of boundary on elementary jump implementations

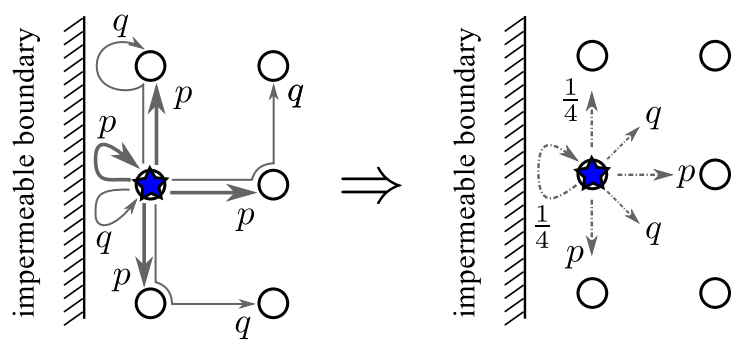

Fig. 5: The analyzed random walks on the square lattice: Diagram and probabilistic weights of the walker jumps near an impermeable boundary.

are treated as the individual implementations of walker elementary motion (Fig. 4, right fragment). In a similar way the other implementations of one elementary step are constructed.

Keeping in mind the example considered above, the term internal causality in the name of these random walks is used to emphasize the fact that the second jump $\mathbf{i}_{-}$is caused by the first one $\mathbf{j}_{+}$. In other words, without the jump $\mathbf{j}_{+}$the jump $\mathbf{i}_{-}$cannot arise but not vice versa. If something, e.g., the system boundary, blocks the walker motion in the direction $\mathbf{i}_{-}$but not in the direction $\mathbf{j}_{+}$the jump $\mathbf{j}_{+}$of the walker is not affected. On the contrary, when something blocks the walker motion in the direction $\mathbf{j}_{+}$the second jump $\mathbf{i}_{-}$cannot arise independently of the fact whether the walker motion in the direction $\mathbf{i}_{-}$is blocked or is not. Therefore only one trajectory joining the start point and the left node (Fig. 4, right fragment) is possible, i.e., it is composed of first $\mathbf{j}_{+}$then $\mathbf{i}_{-}$but not in the opposite order. Figure 5 illustrates this effect of the reflecting (impermeable) boundary on the implementations of one elementary step just near the boundary and their probabilistic weights.

The desired diffusion matrix $\mathbf{D}$ can be introduced via constructing the relationship between the probability (diffusion) flux $\mathbf{Q}$ and the gradient $\nabla G$ of the probability density $G(\mathbf{x}, t)$. Appealing the standard definition 
two components of diffusion flux $\mathbf{Q}$

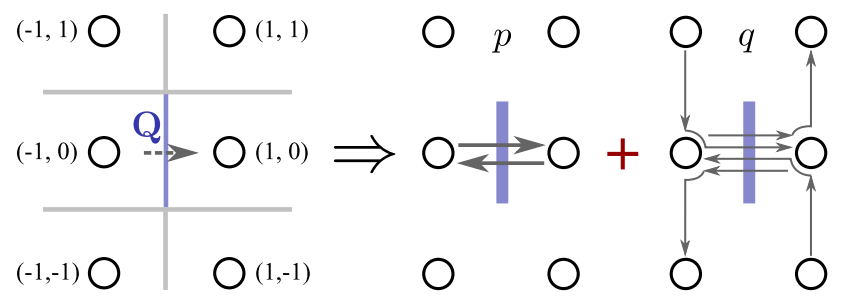

Fig. 6: The diagram illustrating the relationship between the diffusion flux and possible walker transitions.

of diffusion flux the lattice description of the given random walks enables us to calculate it in the following way. First, for the square lattice we construct the set of the Wigner-Seitz cells (Fig. 6) and regard the probability density as the number of walkers $P(\mathbf{x}, t)$ located at a given node $\mathbf{x}$ at time $t$ divided by the "volume" $a^{2}$ of the Wigner-Seitz cell,

$$
G(\mathbf{x}, t)=\frac{1}{a^{2}} P(\mathbf{x}, t) .
$$

Second, taking into account the motion direction, we count the number of walkers $N(t)$ crossing a given face $i$ of the Wigner-Seitz cell during the time $\tau$ via one elementary step. Dividing the result by the face "area" $a$ and the duration of one elementary step $\tau$, we obtain the diffusion flux $Q_{n}$ normal to this face,

$$
Q_{n}(t)=\frac{1}{a \tau} N_{i}(t)
$$

Finally, the obtained value (11) is ascribed to the center point of the face $i$ with orientation specified by the face normal vector. These constructions are illustrated in Fig. 6 and the presented particular example allows us to write

$$
\begin{aligned}
& Q_{x}(0, t)=\frac{a}{\tau}\left\{p\left[G_{-1,0}(t)-G_{1,0}(t)\right]\right. \\
& \left.+q\left[G_{-1,1}(t)+G_{-1,0}(t)-G_{1,0}(t)-G_{1,-1}(t)\right]\right\} .
\end{aligned}
$$

Here without loss of generality the origin is set at the center of the face under consideration. Expression (12) admits the continuous approximation via the Taylor expansion

$$
\begin{aligned}
G_{i, j}=G\left(\mathbf{x}_{i, j}, t\right) & =G\left(\frac{1}{2} a i, a j, t\right) \\
& =i \frac{1}{2} a \partial_{x} G(0, t)+j a \partial_{y} G(0, t),
\end{aligned}
$$

where $i= \pm 1$ and $j=0, \pm 1$. The substitution of (13) into (12) yields

$$
Q_{x}(0, t)=-\frac{(1+\epsilon) a^{2}}{4 \tau} \partial_{x} G(0, t)+\frac{\epsilon a^{2}}{2 \tau} \partial_{y} G(0, t) .
$$

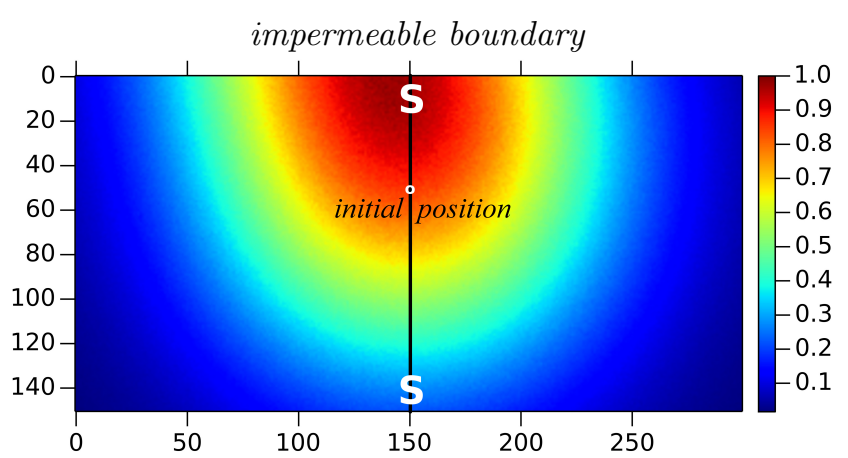

Fig. 7: Distribution function of walkers, the probability density $G(\mathbf{x}, t)$, normalized to its maximum. In numerical simulation the asymmetry parameter $\epsilon=0.2$, the trajectory origin $\left\{x_{0}=150, y_{0}=50\right\}$, and the number of steps in one trajectory $N=3 y_{0}^{2}$ were used. The coordinate labels are the node indices.

In a similar way we get

$$
Q_{y}(0, t)=-\frac{(1+\epsilon) a^{2}}{4 \tau} \partial_{y} G(0, t)-\frac{\epsilon a^{2}}{2 \tau} \partial_{x} G(0, t)
$$

Expressions (14) can be interpreted as the two components of relation (8), where the regular drift velocity $\mathbf{V}=0$ and the desired non-symmetric diffusion matrix $\mathbf{D}$ is specified by the equalities

$$
D_{x x}=D_{y y}=D^{s}, \quad D_{y x}=-D_{x y}=D^{a}=\frac{\epsilon a^{2}}{2 \tau} \text {. }
$$

The non-symmetry of the diffusion matrix must be reflected in the boundary conditions. For example, if the region of random walks is bounded by the impermeable (reflecting) interface $y=0$ then

$$
\left[D^{s} \partial_{y} G+D^{a} \partial_{x} G\right]_{y=0}=0
$$

must hold at this interface. It should cause the asymmetry of the probability density $G(\mathbf{x}, t)$ with respect to the boundary point nearest to the origin of random walks. Numerical simulation justifies this statement (Fig. 7). In fact, if the diffusion matrix were symmetric, so should be the distribution function with respect to the vertical line SS. As seen, the found asymmetry with respect to the line SS is characterized by spatial scales much larger than the lattice spacing. Therefore, the constructed random walks do exhibit non-symmetric properties on scales much larger than the lattice spacing. Thereby, the continuous approximation of the relationship between the diffusion flux and the gradient of the probability distribution function does be based on a non-symmetric tensor.

\section{Conclusions}

A new type non-symmetric diffusion problem has been considered and the corresponding Brownian motion implementing such diffusion processes has been 
constructed. The characteristic feature of these stochastic processes is the non-symmetry of the constant diffusion matrix. The photomagnetoelectric effect is noted in justifying the interest to the given problem.

As an example, random walks with internal causality on a square lattice are studied in detail. By construction, the elementary step of a random walker on the lattice may consist of two succeeding jumps to the nearest neighboring nodes along the $x$ - and then $y$-axis or the $y$ and then $x$-axis ordered, e.g., clock-wise. The causality means that the second fragment of the elementary step is due to the first one, i.e., the second fragment can arise only if the first one has been implemented but not vice versa. In particular, if something blocks the implementation of the second fragment, the first one may be not affected, whereas if it blocks the first fragment, the second one cannot be implemented in any case.

On time scales $t \gg \tau$, where $\tau$ is the duration of one element step, such random walks admit the continuous description. Within this description, the found relationship between the corresponding probability flux and the gradient of the probability density contains the nonsymmetric diffusion matrix; its symmetric component enters the Fokker-Plank equation. The non-symmetry of the diffusion matrix should be reflected in the boundary conditions and the spatial structure of the probability density for these random walks in a bounded region. It is directly demonstrated by numerical simulation.

The constructed random walks are characterized by a certain rotational regularity being responsible for the existence of the antisymmetric component of the diffusion matrix; the translational motion of random walker is purely symmetric, so the regular drift velocity is equal to zero. It enables us we pose a question about the completeness of describing stochastic processes in terms of the Fokker-Planck equation or stochastic differential equations. Indeed, this formalism ignores the internal structure of elementary steps, whereas the given example demonstrates the fact that particular spatial details of the walker motion within one elementary step can affect the macroscopic behavior of diffusion processes.

\section{Acknowledgments}

This work was supported in part by the JSPS Grants-in-Aid for Scientific Research Program, Grant 24540410-0001.

\section{References}

[1] I. Kikoin and M. Noskov: A New Type of Photoelectric Effect in Cuprous Oxide in a Magnetic Field, Nature 131, pp. 725-726, 1933.

[2] I. K. Kikoin and S. D. Lazarev: Photoelectromagnetic Effect, Physics-Uspekhi 21, pp. 297-308, 1978.
[3] W. van Roosbroeck: Theory of the Photomagnetoelectric Effect in Semiconductors, Phys. Rev. 101, pp. 1713-1725, 1956.

[4] F. Effenberger, H. Fichtner, K. Scherer, S. Barra, J. Kleimann, and R. D. Strauss: A Generalized Diffusion Tensor for Fully Anisotropic Diffusion of Energetic Particles in the Heliospheric Magnetic Field, Astrophys. J. 750, p. 108 (8 pages), 2012.

[5] L. Nedžad: Markov Jump Processes Approximating a Nonsymmetric Generalized Diffusion: Numerics Explained to Probabilists, arXiv:0804.0848v4, pp. 1-21, 2010.

[6] J.-D. Deuschel and T. Kumagai: Markov Chain Approximations to Nonsymmetric Diffusions with Bounded Coefficients, Commun. Pure Appl. Math. 66, pp. 821-866, 2013.

[7] I. Lubashevsky: Towards Multi-Dimensional Nonlinear Langevin Equation, in: Proceedings of the 45th ISCIE International Symposium on Stochastic Systems Theory and Its Applications Okinawa, Nov. 1-2, 2013, pp. 278-283, Institute of Systems, Control and Information Engineers (ISCIE), 2013.

[8] Z. Schuss: Theory and Applications of Stochastic Processes: An Analytical Approach, Springer Science+Business Media, LLC, New York, 2009.

[9] C. Gardiner: Stochastic Methods: A Handbook for the Natural and Social Sciences, Springer Verlag, Berlin, 2009, 4th ed.

[10] A. D. Wentzell: On boundary conditions for multidimensional diffusion processes, Theory Prob. Appl. 4, pp. 164-177, 1959.

[11] K.-i. Sato and T. Ueno: Multi-dimensional diffusion and the Markov process on the boundary, $J$. Math. Kyoto Univ. 4, pp. 529-605, 1965.

[12] H. Kunita: General Boundary Conditions for Multi-Dimensional Diffusion Processes, J. Math. Kyoto Univ. 10, pp. 273-335, 1970.

[13] S. Watanabe: On stochastic differential equations for multi-dimensional diffusion processes with boundary conditions, J. Math. Kyoto Univ. 11, pp. 169-180, 1971.

[14] A. V. Skorokhod: Stochastic equations for diffusion processes in a bounded region, Theory of Prob. Appl. 6, pp. 264-274, 1961.

[15] N. Ikeda and S. Watanabe: Stochastic Differential Equations and Diffusion Processes, Kodansha Ltd., Tokyo, 1989, 2nd ed.

[16] R. Mahnke, J. Kaupuzs, and I. Lubashevsky: Physics of Stochastic Processes: How Randomness Acts in Time, WILEY-VCH Verlag GmbH \& Co. KGaA, Weinheim, 2009. 